\title{
Antitumor activity of humanized monoclonal antibody against HM1.24 antigen in human myeloma xenograft models
}

\author{
SHIGETO KAWAI $^{1 *}$, YASUSHI YOSHIMURA ${ }^{*}$, SHIN-ICHIRO IIDA $^{1}$, YASUKO KINOSHITA $^{1}$, \\ YASUO KOISHIHARA ${ }^{1}$, SHUJI OZAKI ${ }^{2}$, TOSHIO MATSUMOTO ${ }^{2}$, \\ MASAAKI KOSAKA $^{3}$ and HISAFUMI YAMADA-OKABE ${ }^{1}$
}

\author{
${ }^{1}$ Pharmaceutical Research Department III, Chugai Pharmaceutical Co., Ltd., 200 Kajiwara, Kamakura, Kanagawa 247-8530; \\ ${ }^{2}$ Department of Medicine and Bioregulatory Sciences, Institute of Health Biosciences, The University of Tokushima \\ Graduate School and Division of Transfusion Medicine, Tokushima University Hospital, Tokushima 770-8501; \\ ${ }^{3}$ Tokushima Red Cross Blood Center, Tokushima 770-0044, Japan
}

Received August 17, 2005; Accepted October 5, 2005

\begin{abstract}
A humanized monoclonal antibody against HM1.24 antigen (AHM), which is highly expressed on multiple myeloma (MM) cells, induced antibody-dependent cellular cytotoxicity (ADCC) in vitro. In this study, we further characterized AHM and evaluated its potency for clinical application. AHM bound to HM1.24 antigen with a dissociation constant of $0.35 \mathrm{nM}$, and its epitope resided between Leu $^{116}$ and $\mathrm{Leu}^{127}$ of the HM1.24 antigen. Single intravenous injection of AHM significantly inhibited tumor growth in both orthotopic and ectopic human MM xenograft models. AHM reduced serum $M$ protein levels and prolonged survival of mice intravenously inoculated with KPMM2 and ARH-77 cells. The number of KPMM2 cells in bone marrow or tumor volume of subcutaneously inoculated RPMI 8226 cells was also inhibited by AHM. The antitumor activity of AHM against tumor cells in bone marrow was diminished when the mice were pretreated with anti-Fc $\gamma$ receptor III/II antibody, demonstrating that antitumor activity by AHM requires effector cell functions in vivo. Experiments involving in vitro ADCC assays indicated that NK cells and monocytes/macrophages serve as effector cells for AHM-induced ADCC in mouse and human. Thus, AHM will provide an additional treatment option for MM.
\end{abstract}

\section{Introduction}

Patients with multiple myeloma (MM), caused by clonal expansion of plasma cells and subsequent invasion of the tumor

Correspondence to: Dr Hisafumi Yamada-Okabe, Pharmaceutical Research Department III, Chugai Pharmaceutical Co., Ltd., 200 Kajiwara, Kamakura, Kanagawa 247-8530, Japan

E-mail: okabehsf@chugai-pharm.co.jp

${ }^{*}$ Contributed equally

Key words: HM1.24, humanized antibody, antibody-dependent cellular cytotoxicity, multiple myeloma, KPMM2, ARH-77, RPMI 8226, xenograft model cells into bone marrow, suffer from severe anemia, humoral immunodeficiency and concomitant microbial infections (1). In addition, MM patients often show elevated blood levels of interleukin-6 (IL-6), IL-1ß and tumor necrosis factor $\alpha$. Because these cytokines enhance osteolysis, patients suffer from bone pain, fractures and hypercalcemia (2). Recently, new therapies such as thalidomide and Velcade have been used in clinic and have shown improved antitumor effect against MM. However, efficacy of these drugs is still not satisfactory, and they can cause adverse effects such as peripheral neuropathy, which limits the full-dose combination with other chemotherapeutic agents (3-5). Therefore, development of potent and safe new drugs is required.

Previously, we generated a monoclonal antibody (mAb) against the HM1.24 antigen (also named CD317) that is highly expressed in terminally differentiated normal and neoplastic B cells $(6,7)$. The mouse mAb against HM1.24 (mAHM) was effective in human MM xenograft models (8). In addition, we created a humanized anti-HM1.24 mAb (AHM) and found that it induced antibody-dependent cellular cytotoxicity (ADCC) against human MM cell lines (9). Although AHM induced complement-dependent cytotoxicity (CDC) in the presence of baby rabbit complements, no CDC occurred in the presence of human or mouse serum (10). Therefore, AHM is considered to elicit antitumor activity through ADCC both in mouse and human.

In this study, we further characterized AHM and evaluated its potency for clinical applications. AHM bound to HM1.24 antigen with a similar dissociation constant $(\mathrm{Kd})$ to that of mAHM, and showed significant tumor growth inhibition and prolonged survival in human MM xenograft models in a manner dependent on effector cell functions. Furthermore, NK cells and monocytes/macrophages are expected to serve as effector cells both in mouse and human.

\section{Materials and methods}

Antibodies. A mouse mAb carrying mouse IgG2aк (mAHM) and a humanized mAb carrying the human $\operatorname{IgG} 1 \kappa$ (AHM) against the HM1.24 antigen were prepared as reported 
previously $(9,10)$. Mouse IgG2a (UPC10; Biogenesis, Kingston, $\mathrm{NH}$ ) and human IgG1к (hIgG1; Sigma, St. Louis, MO) were used as control $\mathrm{IgG}$.

Fluorescein-labeled mAHM (mAHM-FITC) and mouse IgG2a (mIgG2a-FITC) were generated by labeling mAHM and UPC10 with NHS-fluorescein (Pierce, Rockford, IL) and purified by gel filtration with PD-10 Sephadex G-25 M column (Amersham Bioscience, Tokyo, Japan). ${ }^{125}$ I-labeled AHM ( $\left.{ }^{125} \mathrm{I}-\mathrm{AHM}\right)$ was created by radiolabeling $\mathrm{AHM}$ with $\mathrm{Na}^{125} \mathrm{I}$ by the Tejedor method (11) and purified by affinity chromatography with HiTrap Protein A column (Amersham Bioscience) followed by gel filtration chromatography with Superdex 200HR 10/30 (Amersham Bioscience). The specific activity of ${ }^{125} \mathrm{I}-\mathrm{AHM}$ was $1.12 \mathrm{MBq} / \mu \mathrm{g}$.

Cells. Cells of the human MM cell line KPMM2 (12) were cultured in RPMI-1640 medium (Invitrogen, Carlsbad, CA) supplemented with $20 \%$ fetal bovine serum (FBS) and $4 \mathrm{ng}$ / ml IL-6 (PeproTech, Rocky Hill, NJ). Cells of the human B-lymphoblast cell line ARH-77 and MM cell line RPMI 8226 were cultured in RPMI-1640 medium supplemented with $10 \%$ FBS. For in vivo passage of KPMM2, small pieces $\left(27 \mathrm{~mm}^{3}\right)$ of KPMM2 tumor were subcutaneously transplanted into severe combined immunodeficiency (SCID) mice (CB17/IcrPrkdc ${ }^{\text {scid }}$, Clea Japan, Tokyo, Japan).

Flow cytometry. Cell surface expression of the HM1.24 antigen was analyzed by flow cytometry. Cells $\left(5 \times 10^{5}\right)$ were incubated with $40 \mu \mathrm{g} / \mathrm{ml} \mathrm{mAHM}$-FITC or mIgG2a-FITC in phosphate buffered saline (PBS) containing $0.1 \%$ bovine serum albumin and $0.1 \%$ sodium azide (FCM/PBS) for 30 min on ice. Cells were then washed twice with FCM/PBS and analyzed using an EPICS XL flow cytometer (Beckman Coulter, Tokyo, Japan).

Antibody binding assay. Cells $\left(10^{6}\right.$ cells $\left./ \mathrm{ml}\right)$ were incubated with $0.125 \mathrm{nM}{ }^{125} \mathrm{I}-\mathrm{AHM}$ and serial dilutions of non-radiolabeled AHM for $3 \mathrm{~h}$ on ice. Then, the cells were gently applied onto FBS, separated from the supernatants by centrifugation at $11,000 \mathrm{x} \mathrm{g}$ for $3 \mathrm{~min}$, and frozen at $-80^{\circ} \mathrm{C}$. The resulting frozen cell pellets and supernatants were isolated by cutting the sample tubes and radioactivity was quantified by a COBRA II gamma counter (Packard Instrument Company, Meriden, CT). The Kd of AHM and the number of AHM bound to the cells was determined by Scatchard analysis. Radioactivity detected in the cells even in the presence of a 1,000-fold excess of non-radiolabeled AHM was considered non-specific binding.

Epitope mapping. cDNA for the HM1.24 antigen with Cterminal and $\mathrm{N}$-terminal deletions were cloned at the EcoRI/ NotI site of glutathione S-transferase (GST) expression vector pGEX-4T-3 (Amersham Bioscience). After transformation of E. coli DH5 $\alpha$ with the resulting plasmid DNA, HM1.24 expression was induced by isopropyl $\beta$-(D)-thiogalactopyranoside (Wako Pure Chemical Industries, Osaka, Japan). The GST-HM1.24 fusion proteins were separated on a 4-20\% gradient SDS-polyacrylamide gel, transferred onto PVDF membranes (Tefco, Tokyo, Japan), and hybridized with $1 \mu \mathrm{g}$ / ml AHM, hIgG1 or anti-GST antibody (Amersham Bioscience). After incubation with alkaline phosphatase-labeled anti-human IgG antibody (Biosource International, Camarillo, CA) or alkaline phosphatase-labeled anti-goat IgG antibody (Zymed Laboratories, South San Francisco, CA), the GSTHM1.24 fusion proteins were visualized by incubation in a colorimetric solution containing $100 \mathrm{mM}$ Tris- $\mathrm{HCl}$ ( $\mathrm{pH} 9.5)$, $100 \mathrm{mM} \mathrm{NaCl}, 5 \mathrm{mM} \mathrm{MgCl} 2,300 \mu \mathrm{g} / \mathrm{ml}$ nitroblue tetrazolium and $165 \mu \mathrm{g} / \mathrm{ml}$ 5-bromo-4-chloro-3-indolyl phosphate.

Animals. Six- to eight-week-old male SCID mice were injected subcutaneously with $20 \mu 1$ of rabbit anti-asialo GM1 antiserum (Wako Pure Chemical Industries) a day before tumor inoculation to eradicate residual NK cells. Day 0 represents the day when the mice were inoculated with the indicated tumor cells. The animals used in the experiments were treated in accordance with Chugai Pharmaceutical's ethical guidelines of animal care, handling and termination.

Determination of antitumor activities. KPMM2 xenograft model: $6 \times 10^{6}$ cells/mouse of in vivo-passaged KPMM2 cells were intravenously injected into SCID mice. The mice were divided into 5 groups on day 9 based on serum human $\mathrm{IgG}$ concentration (M protein), determined by enzyme-linked immunosorbent assay (ELISA) as described previously (13). AHM (4 or $20 \mu \mathrm{g} /$ mouse) or hIgG1 (20 $\mu \mathrm{g} /$ mouse) was administered intravenously on day 10 . Each group consisted of 8 mice except for the hIgG1 group (7 mice). Melphalan (Sigma) was suspended in $0.2 \%$ carbomethylcellulose solution and was administered orally at doses of 1 or $3 \mathrm{mg} / \mathrm{kg}$ every day from day 10 to day 14 .

To examine the effects of AHM on the tumor cells in bone marrow, mice were administered $1 \mathrm{mg} / \mathrm{kg}$ of AHM or vehicle on day 12 , and bone marrow cells were collected from the femur $0,6,24$ or $48 \mathrm{~h}$ after the administration. After incubation with PE-labeled anti-CD38 mAb (Becton-Dickinson, Franklin Lakes, NJ) or control PE-labeled mouse IgG1 (BectonDickinson) on ice for $30 \mathrm{~min}, \mathrm{CD} 38$ positive tumor cells in the bone marrow were analyzed by FACScan flow cytometer (Becton-Dickinson). To examine the involvement of $\mathrm{Fc} \gamma$ receptors $(\mathrm{Fc} \gamma \mathrm{Rs})$ in antitumor activity of AHM, mice were administered $250 \mu \mathrm{g} /$ mouse of anti-mouse Fc $\gamma$ RIII/II mAb (BD Pharmingen, Franklin Lakes, NJ) a day before the administration of AHM or vehicle (day 13). Tumor cells in the bone marrow were analyzed $48 \mathrm{~h}$ after the administration of AHM or vehicle control.

ARH-77 xenograft model: $6 \times 10^{6}$ cells/mouse of ARH-77 cells was intravenously injected into SCID mice. The mice were divided into 3 groups on day 9 based on the serum $M$ protein levels. Each group consisted of 8 mice. AHM (20 or $100 \mu \mathrm{g} /$ mouse) or hIgG1 (100 $\mu \mathrm{g} /$ mouse) was administered intravenously on day 10 .

RPMI 8226 xenograft model: $6 \times 10^{6}$ cells/mouse of RPMI 8226 cells was subcutaneously injected into SCID mice. Mice were divided into 3 groups based on tumor volume on day 17 when the average tumor volume reached $100 \mathrm{~mm}^{3}$. Each group consisted of 10 mice, and AHM ( 0.25 or $1 \mathrm{mg} / \mathrm{kg})$ or vehicle was administered intravenously on day 17 . Tumor volume was measured once a week and tumor weight was measured on day 45. Tumor volume was determined with the formula: $a b^{2} / 2$ $\left(\mathrm{mm}^{3}\right)$, where $a$ and $b$ are the longest and shortest diameters, respectively. 
(A)

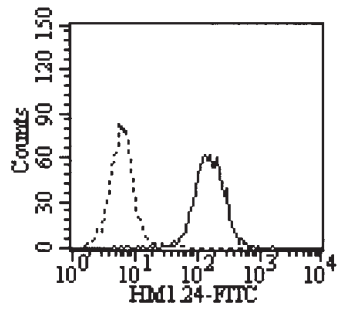

(D)

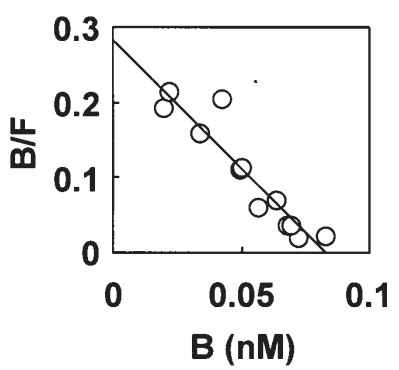

(B)

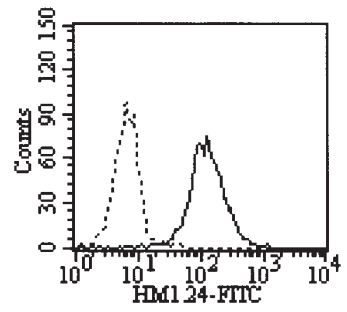

(E)

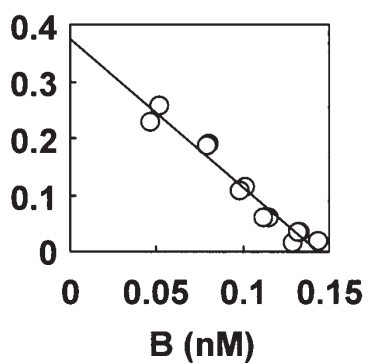

(C)

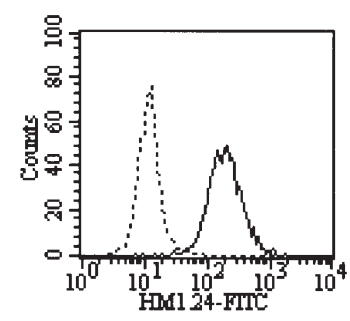

(F)

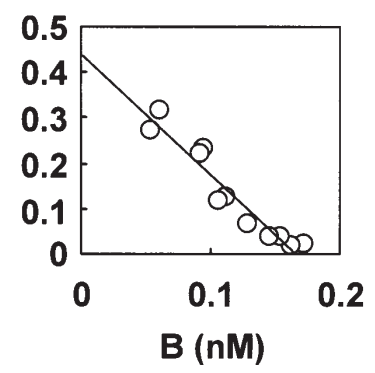

Figure 1. Affinity of AHM to HM1.24 antigen. Expression of HM1.24 antigen on cell lines was analyzed by flow cytometry and ${ }^{125}$ I-AHM binding assay. KPMM2 (A), ARH-77 (B) or RPMI 8226 (C) was stained with mAHM-FITC ( - ) or mIgG2a-FITC (------) and analyzed by flow cytometry. ${ }^{125}$ I-AHM binding assays were carried out twice, and the representative Scatchard plots for KPMM2 (D), ARH-77 (E) and RPMI 8226 (F) are shown.

Preparation of effector cells. Peripheral blood was obtained from healthy volunteers after approval by the Ethics Committee of Chugai Pharmaceutical Co., Ltd. Peripheral blood mononuclear cells (PBMCs) were separated by gradient centrifugation with Ficoll-Paque (Amersham Bioscience). NK cells were depleted from PBMCs by magnetic cell sorting with CD56 MicroBeads (Miltenyi Biotec, Bergisch Gladbach, Germany). NK cells and monocytes were purified from PBMCs by magnetic cell sorting with NK Cell Isolation Kit and Monocyte Isolation Kit (Miltenyi Biotec), respectively.

Spleen cells were obtained from SCID mice. NK cells were depleted by administration of rabbit anti-asialo GM1 antiserum 1 day before harvesting effector cells in some of the mice and macrophages were obtained by rinsing the peritoneal cavity of these mice. Purified macrophages were positive for F4/80 (macrophage marker) but negative for DX5 (pan-NK cell marker) or Gr-1 (neutrophil marker) and did not show natural killer activity against NK-sensitive YAC- 1 cells. The mAbs for the flow cytometry were purchased from BD Pharmingen except for F4/80 (Cederlane, Ontario, Canada).

Determination of ADCC activities. ADCC activity of AHM was determined by ${ }^{51} \mathrm{Cr}$ release assay. Target cells were labeled with $150 \mu \mathrm{Ci}\left(5.55 \mathrm{MBq}\right.$ ) of ${ }^{51} \mathrm{Cr}$-sodium chromate (Amersham Pharmacia) at $37^{\circ} \mathrm{C}$ for $1 \mathrm{~h}$. The cells were then washed three times with RPMI-1640 medium supplemented with 10\% FBS and suspended in the same medium. The radiolabeled cells were plated on 96 -well round-bottomed plates $\left(10^{4}\right.$ cells/well $)$ and incubated with various concentrations of AHM or hIgG1 on ice for 15 min. In some experiments, anti-human Fc $\gamma$ RIII $\mathrm{F}\left(\mathrm{ab}^{\prime}\right)_{2}$ mAb (Medarex, Annandale, NJ), anti-human Fc $\gamma$ RII Fab mAb (Medarex), anti-human Fc $\gamma \mathrm{RI} F\left(\mathrm{ab}^{\prime}\right)_{2} \mathrm{mAb}$ (Ancell, Bayport, MN), or anti-mouse Fc $\gamma$ RIII/II mAb were added to the culture at a concentration of 10 or $20 \mu \mathrm{g} / \mathrm{ml}$. Effector cells were then added to the cells at various effector-to-target (E:T) ratios, and the cells were further incubated for 4 or $8 \mathrm{~h}$. One hundred microliters of the supernatant from each well was collected and radioactivity in the supernatants was measured using a gamma counter. Radioactivity in the supernatants of the ${ }^{51} \mathrm{Cr}$-labeled cells incubated without effector cells and antibodies were considered spontaneous ${ }^{51} \mathrm{Cr}$ release; those of the cells after incubation in 1\% NP-40 solution were considered maximum ${ }^{51} \mathrm{Cr}$ release. Cytotoxicity (\%) was determined with the formula: $(A-C) /(B-C) \times 100$ where $A, B$ and $C$ represent ${ }^{51} \mathrm{Cr}$ release in each experiment, maximum ${ }^{51} \mathrm{Cr}$ release, and spontaneous ${ }^{51} \mathrm{Cr}$ release, respectively. All experiments were done in triplicate and values in the figures are means + SD.

Statistical analysis. Survival of the mice, $M$ protein, and tumor weight were analyzed by Dunnett's test, and differences with $\mathrm{P}$-values of $<0.05$ were considered significant.

\section{Results and Discussion}

Antigen binding and epitope of AHM. First, we determined the affinity of AHM to HM1.24 antigen by flow cytometry and ${ }^{125} \mathrm{I}$-AHM binding assay (Fig. 1). The Kd of AHM to the antigen was $0.346 \pm 0.055 \mathrm{nM}$ (mean $+\mathrm{SD}, \mathrm{n}=6)$, which was very similar to that of mAHM (1.09 nM) (7). AHM was reported to bind cell surface HM1.24 antigen and kill target cells by ADCC (9), but the epitope of AHM remains to be clarified. Therefore, we mapped the epitope of AHM by Western blotting using GST fusion protein carrying various parts of HM1.24 antigen. AHM bound to C-terminally deleted HM127 encompassing Val ${ }^{35}-$ Leu $^{127}$ but not to HM126 encompassing $\mathrm{Val}^{35}$-Lys ${ }^{126}$, indicating that the epitope is 
(A)
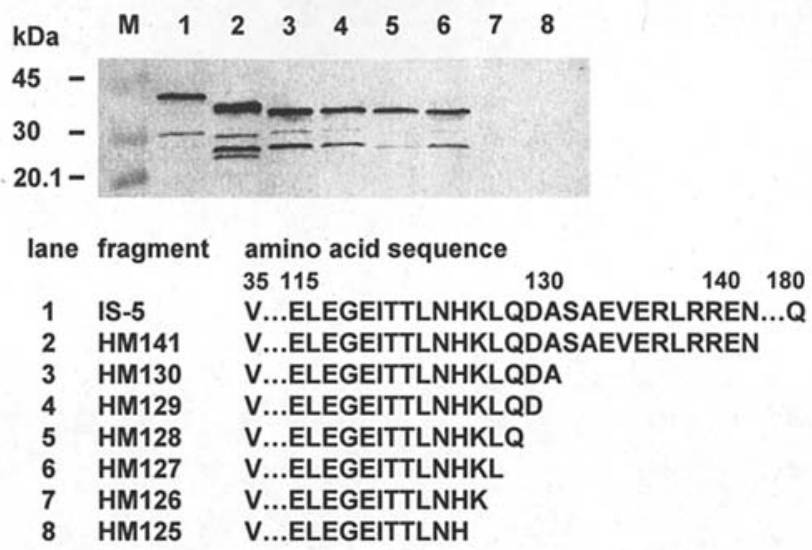

(B)

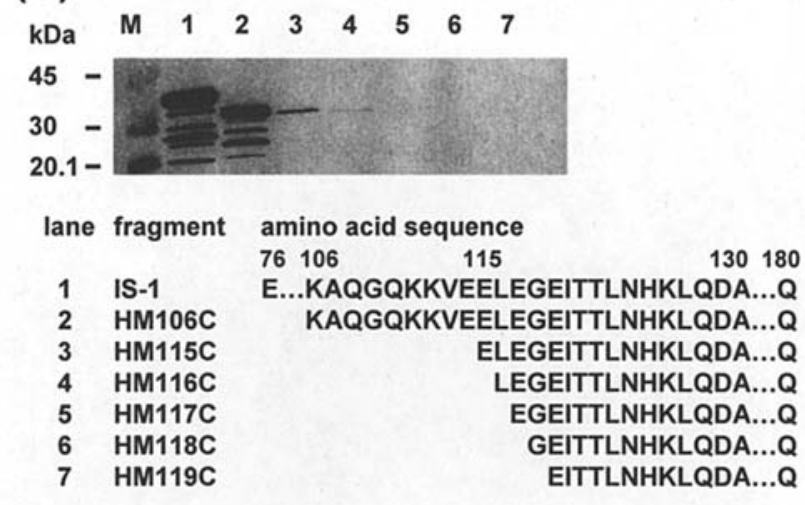

(C)

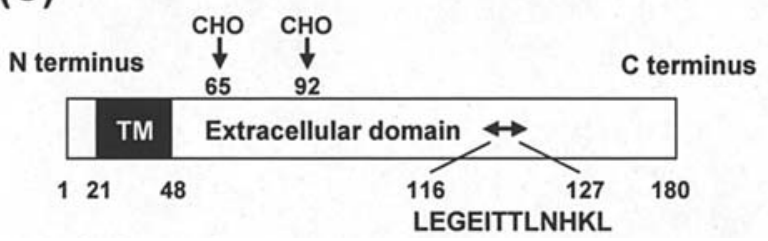

Figure 2. Epitope of AHM in the HM1.24 antigen. C-terminally (A) and $\mathrm{N}$-terminally (B) deleted fragments of HM1.24 were expressed in E. coli as the fusion proteins with GST, and the cell lysates were fractionated by SDSPAGE. Binding of AHM to these fragments was determined by Western blot analysis with AHM. Amino acid sequence of each fragment is shown. (C), Amino acid sequence of epitope and its location within HM1.24 antigen is shown. TM, transmembrane region; $\mathrm{CHO}$, putative glycosylation site; LEGEITTLNHKL, amino acid sequence of the epitope.

present between $\mathrm{Val}^{35}$ and Leu ${ }^{127}$ (Fig. 2A). On the other hand, AHM bound to N-terminally deleted HM116C encompassing $\mathrm{Leu}^{116}-\mathrm{Gln}^{180}$ but not to HM117C encompassing Glu ${ }^{117}-\mathrm{Gln}^{180}$, indicating that the epitope locates between $\mathrm{Leu}^{116}$ and Gln ${ }^{180}$ (Fig. 2B). Therefore, the epitope of AHM resides between Leu ${ }^{116}$ and $\mathrm{Leu}^{127}$ of the HM1.24 antigen (Fig. 2C).

Antitumor activity of AHM in mouse xenograft models. As mentioned above, AHM killed HM1.24 antigen-positive human MM cells through ADCC in the presence of human effector cells (9). Therefore, we evaluated the antitumor activities of AHM in orthotopic and ectopic tumor xenograft models using KPMM2, RPMI 8226 and ARH-77 cells. Amounts of HM1.24
(A)

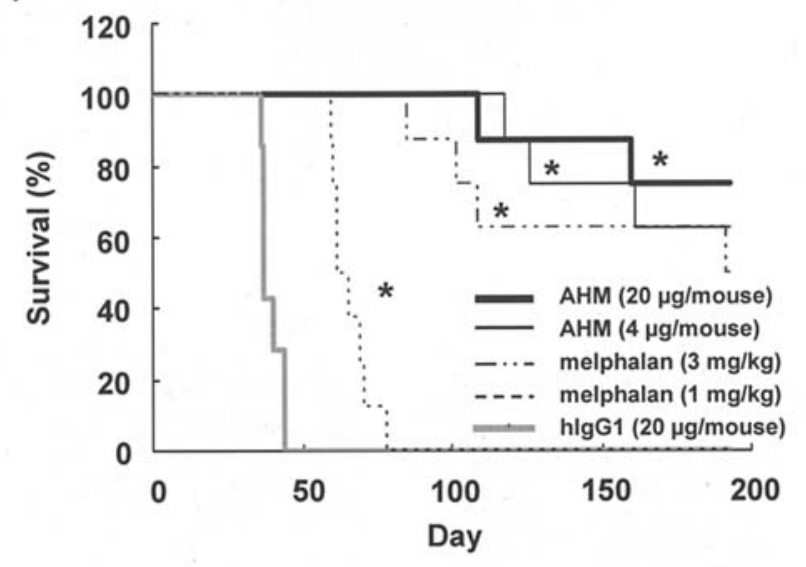

(B)

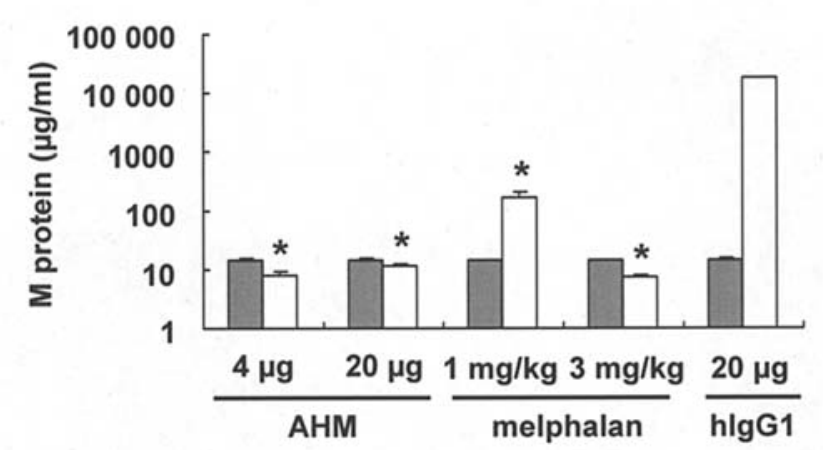

(C)

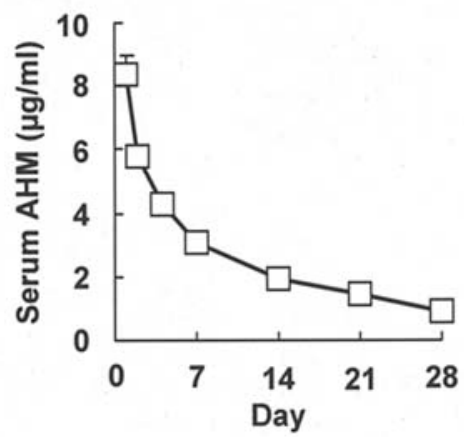

Figure 3. Antitumor activity of AHM in KPMM2 xenograft model. (A), SCID mice were intravenously injected with $6 \times 10^{6}$ cells/mouse of KPMM2 cells. Then, they were intravenously administered 4 or $20 \mu \mathrm{g} /$ mouse of AHM or $20 \mu \mathrm{g} /$ mouse of the control hIgG1 on day 10 , or orally administered 1 or $3 \mathrm{mg} /$ $\mathrm{kg}$ melphalan every day from day 10 to day 14 . Each group consisted of 8 (for AHM and melphalan treated groups) or 7 (for hIgG1 treated group) mice. Percentage of the survived mice is shown. (B), Mice were treated as in (A), and serum levels of $\mathrm{M}$ protein on day 9 (grey) and day 35 (white) were determined by ELISA. Data represent means + SE. (C), SCID mice that had been intravenously injected with $6 \times 10^{6}$ cells/mouse of KPMM2 cells were administered $20 \mu \mathrm{g}$ /mouse of AHM on day 10, and serum concentrations of AHM on the indicated days were determined by ELISA. Data represent means + SE obtained from 5 mice. Asterisks indicate significant difference between the test group and the control group $(\mathrm{p}<0.05$ by Dunnett's test). Day 0 represents the day when the mice were inoculated with tumors.

antigen on KPMM2, RPMI 8226 and ARH-77 cells were estimated by Scatchard analysis as $5.97 \times 10^{4}, 8.72 \times 10^{4}$ and $8.47 \times 10^{4}$ molecules/cell, respectively. Mice intravenously injected with KPMM2 showed tumor masses in bone marrow, osteolytic lesions, hypercalcemia and increased serum M protein (13), and all mice died before day 44 (Fig. 3A). 
(A)

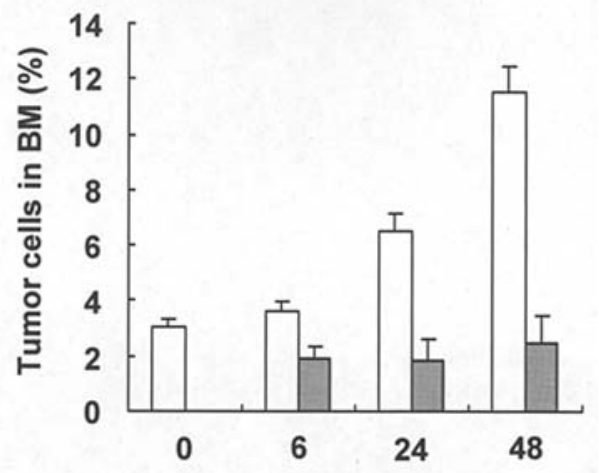

Time after AHM administration (h)

(B)

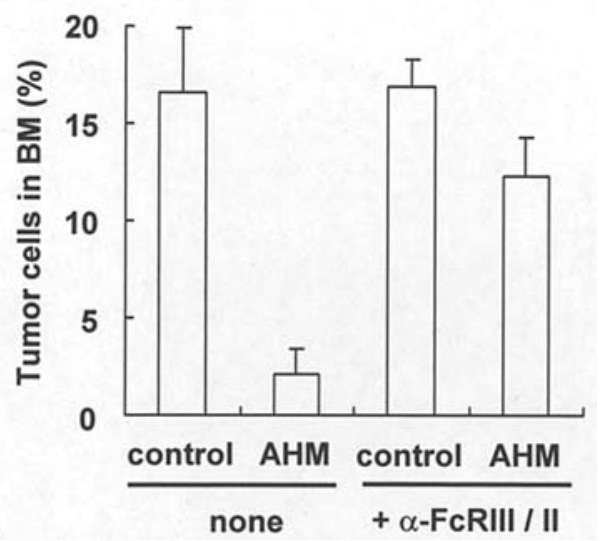

Figure 4. Effect of AHM on tumor cell growth in bone marrow. (A), SCID mice were intravenously injected with $6 \times 10^{6}$ cells/mouse of KPMM2 cells, and they were administered $1 \mathrm{mg} / \mathrm{kg}$ AHM (grey) or PBS (white) on day 12 . KPMM2 cells in bone marrow were collected at the indicated time after the administration of AHM, and were analyzed by flow cytometry after staining with PE-labeled anti-CD38 antibody. Percentage of KPMM2 cells in bone marrow is shown. (B), Mice that had been injected with $6 \times 10^{6}$ cells/mouse of KPMM2 cells were administered $250 \mu \mathrm{g}$ /mouse of anti-Fc $\gamma$ RIII/II mAb on day 13 and then $1 \mathrm{mg} / \mathrm{kg}$ of AHM on day 14. Control mice received PBS. Percentages of KPMM 2 cells in bone marrow were determined $48 \mathrm{~h}$ after administration of AHM. Data represent means + SE obtained from 4 mice. Day 0 represent the day when the mice were inoculated with tumors.

Administration of AHM (4 or $20 \mu \mathrm{g} /$ mouse) on day 10 markedly decreased serum $M$ protein levels and prolonged the survival of the mice as compared to those receiving the control antibody. In addition, the antitumor activity of AHM as revealed by serum $\mathrm{M}$ protein and survival was almost equivalent to that of $3 \mathrm{mg} / \mathrm{kg}$ melphalan (Fig. 3A and B). In KPMM2-bearing mice, plasma half-life and serum concentration of AHM 21-28 days after administration of $20 \mu \mathrm{g} /$ mouse AHM were about 8 days and approximately $1 \mu \mathrm{g} / \mathrm{ml}$, respectively (Fig. 3C). Because $1 \mu \mathrm{g} / \mathrm{ml}$ of AHM was sufficient for the maximum ADCC against KPMM2 cells in vitro, doses should be set to maintain $1 \mu \mathrm{g} / \mathrm{ml}$ or higher concentrations of AHM in serum.

We also examined whether AHM impaired the growth of tumor cells in bone marrow. Administration of AHM (1 mg/ $\mathrm{kg}$ ) on day 12 suppressed the growth of KPMM2 cells in the bone marrow (Fig. 4A), which was diminished by admini-
(A)

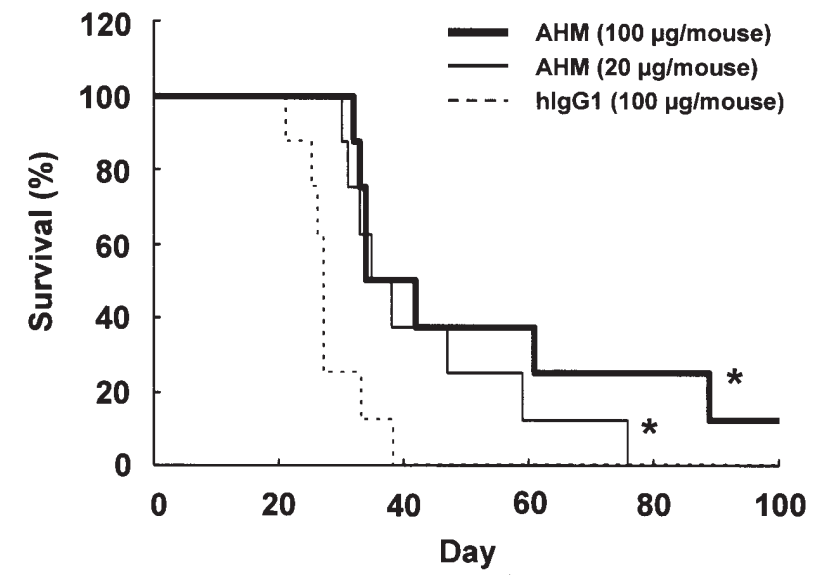

(B)

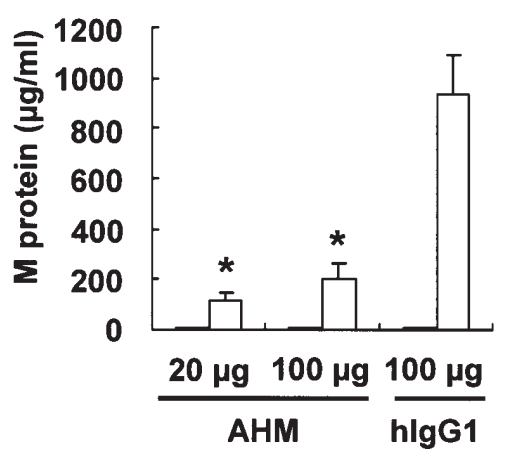

Figure 5. Antitumor activity of AHM in ARH-77 xenograft model. (A), SCID mice were intravenously injected with $6 \times 10^{6}$ cells/mouse of ARH-77 cells. Then, they were intravenously administered 20 or $100 \mu \mathrm{g} /$ mouse of AHM or $100 \mu \mathrm{g} /$ mouse of control hIgG1 on day 10 . Each group consisted of 8 mice. Percentage of the survived mice is shown. (B), Mice were treated as in (A), and serum levels of M protein on day 9 (grey) and day 21 (white) were determined by ELISA. Data represent means + SE obtained from 8 mice. Asterisks indicate significant difference between the test group and the control group $(\mathrm{p}<0.05$ by Dunnett's test). Day 0 represents the day when the mice were inoculated with tumors.

stration of anti-mouse Fc $\gamma$ RIII/II mAb (Fig. 4B). The results indicate AHM affects the growth of tumor cells in bone marrow and that the effect is mediated by effector cell functions.

Antitumor activity of AHM was also examined in two other xenograft models. Mice intravenously inoculated with ARH-77 developed tumors not only in bone marrow but also in lymph nodes, especially around the neck, adrenal gland or intestine, thereby representing highly metastatic states of cancer. Even in such xenograft model, single administration of 20 or $100 \mu \mathrm{g} /$ mouse AHM on day 10 suppressed the serum M protein levels and prolonged the survival as compared to the mice receiving the control antibody (Fig. 5). Tumor growth inhibition by AHM was also evident in the ectopic model. Administration of 0.25 or $1 \mathrm{mg} / \mathrm{kg}$ AHM on day 17 to mice subcutaneously inoculated with RPMI 8226 significantly inhibited tumor growth as evidenced by tumor volume and tumor weight (Fig. 6).

Effector cells for the ADCC activity of AHM. Since antitumor effects by AHM are mediated by effector cell functions (9), 
(A)

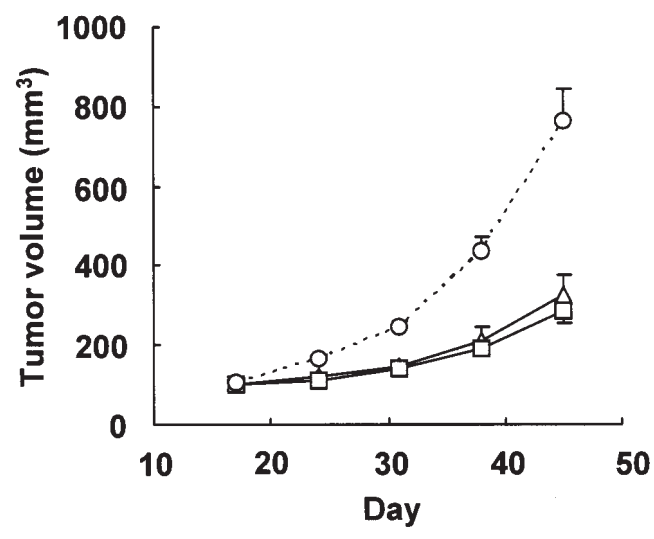

(B)

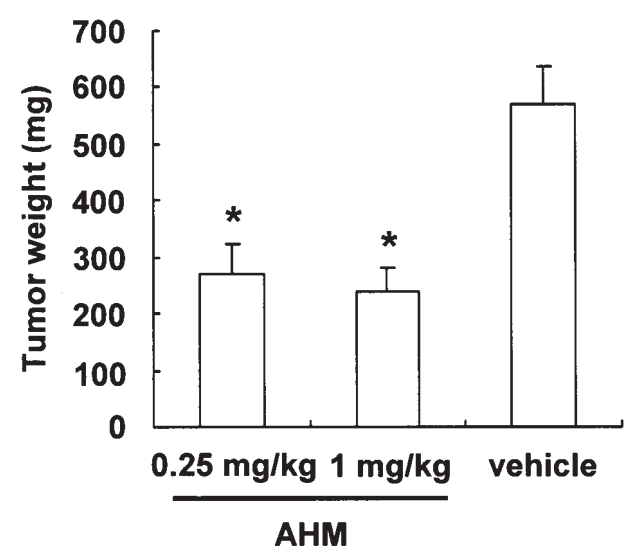

Figure 6. Antitumor activity of AHM in RPMI 8226 xenograft model. SCID mice were subcutaneously inoculated with $6 \times 10^{6}$ cells/mouse of RPMI 8226 cells. Then, they were intravenously administered 0.25 or $1 \mathrm{mg} / \mathrm{kg}$ of AHM or PBS on day 17. Tumor volume was measured once a week (A), and tumor weight was measured on day 45 (B). Each group consisted of 10 mice. Asterisks indicate significant difference between the test group and the control group ( $\mathrm{p}<0.05$ by Dunnett's test). Day 0 represents the day when the mice were inoculated with tumors. $\square$, AHM $(1 \mathrm{mg} / \mathrm{kg}) ; \triangle$, AHM $(0.25 \mathrm{mg} / \mathrm{kg}) ;$, PBS.

we asked which effector cells are responsible for the antitumor activity of AHM. As shown in Fig. 7A, AHM induced ADCC against ARH-77, and to a much lesser extent against KPMM2, with murine spleen cells. ADCC activity was completely diminished in spleen cells collected from mice in which NK cells were depleted. Strong ADCC activity against ARH-77 and KPMM2 was observed with macrophages isolated from the peritoneal cavity of mouse, and the ADCC was nearly completely inhibited by anti-mouse Fc $\gamma$ RIII/II mAb (Fig. 7B). The results indicate that in mouse, macrophages and NK cells mediate the ADCC activity of AHM.

We also asked which types of effector cells mediate the antitumor activity of AHM in human. AHM induced ADCC against KPMM2 in the presence of human PBMCs, whereas PBMC after depletion of NK cells had no effect (Fig. 8A). Strong ADCC activity was also reproduced with purified NK cells but was nearly completely inhibited by anti-Fc $\gamma$ RIII mAb (Fig. 8B). Although much weaker than observed with NK cells,
(A)

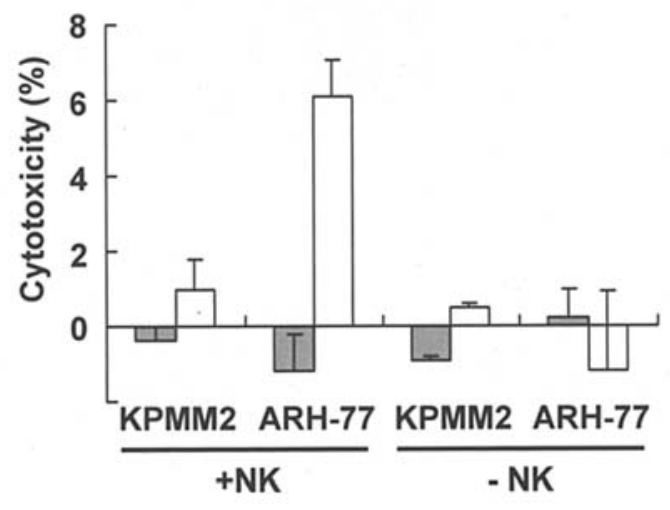

(B)

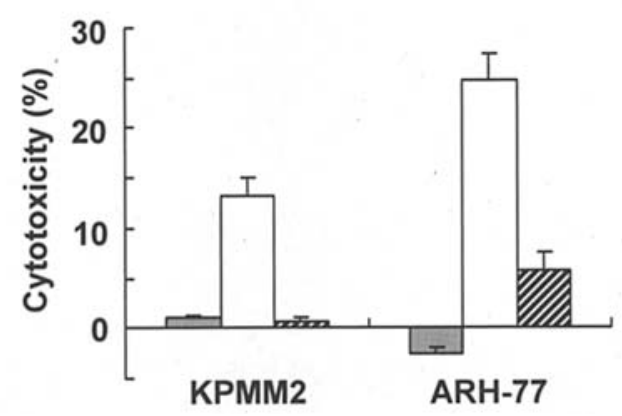

Figure 7. ADCC activity of AHM mediated by murine effector cells. (A), Spleen cells were collected from SCID mice. To deplete NK cells, mice were administered rabbit anti-asialo GM1 antiserum 1 day before harvesting spleen cells. KPMM2 or ARH-77 cells that had been labeled with ${ }^{51} \mathrm{Cr}$ were incubated with spleen cells containing $(+\mathrm{NK})$ or not containing $(-\mathrm{NK}) \mathrm{NK}$ cells for $4 \mathrm{~h}$ at an E:T ratio of 60 in the presence of $1 \mu \mathrm{g} / \mathrm{ml} \mathrm{AHM} \mathrm{(white)} \mathrm{or}$ hIgG1 (grey). (B), Macrophage was collected from peritoneal cavity of SCID mice that had been administered rabbit anti-asialo GM1 antiserum 1 day before harvest. KPMM2 and ARH-77 cells that had been labeled with ${ }^{51} \mathrm{Cr}$ were incubated with macrophages for $8 \mathrm{~h}$ at an E:T ratio of 50 in the presence of $1 \mu \mathrm{g} / \mathrm{ml} \mathrm{AHM} \mathrm{(white)} \mathrm{or} \mathrm{hIgG1} \mathrm{(grey)} \mathrm{together} \mathrm{with} \mathrm{or} \mathrm{without} 20 \mu \mathrm{g}$ / $\mathrm{ml} \alpha-\mathrm{Fc} \gamma \mathrm{RIII} / \mathrm{II} \mathrm{mAb}$ (hatched).

monocytes purified from PBMCs also induced the ADCC activity of AHM which was nearly completely inhibited by the combination of anti-Fc $\gamma$ RIII and anti-Fc $\gamma$ RII mAbs (Fig. 8C). We also examined ADCC activity with neutrophil collected from peripheral blood using density centrifugation and found that purified neutrophil did not mediate ADCC activity of AHM (data not shown). These results suggest that in human, NK cells, and to a lesser extent monocytes, serve as effector cells for the ADCC activity of AHM, which is consistent with our previous observation that the ADCC activity of AHM correlates with the number of NK cells in PBMC (10).

Recently, thalidomide and Velcade have been shown to be effective in the treatment of MM in clinic (3-5). Nevertheless, efficacy of these drugs is still not satisfactory, and these drugs cannot be easily combined with other drugs due to their toxicity. In a phase I/II study, AHM did not cause any serious toxicity when administered to patients with relapsed or refractory MM (Powles R, et al, Japanese Multiple Myeloma 


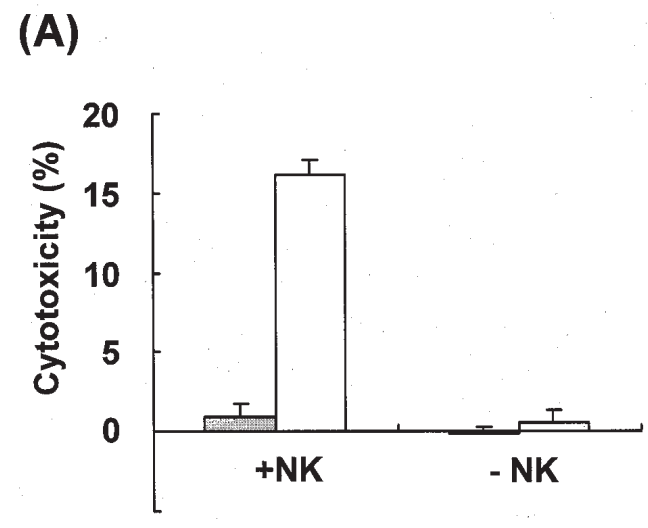

(B)

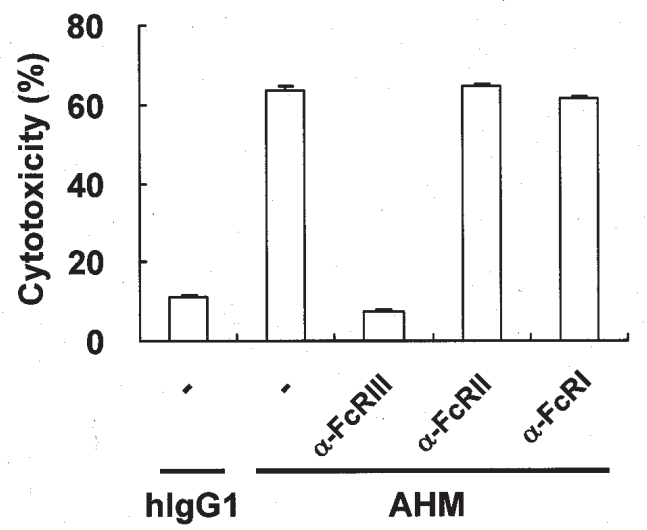

(C)

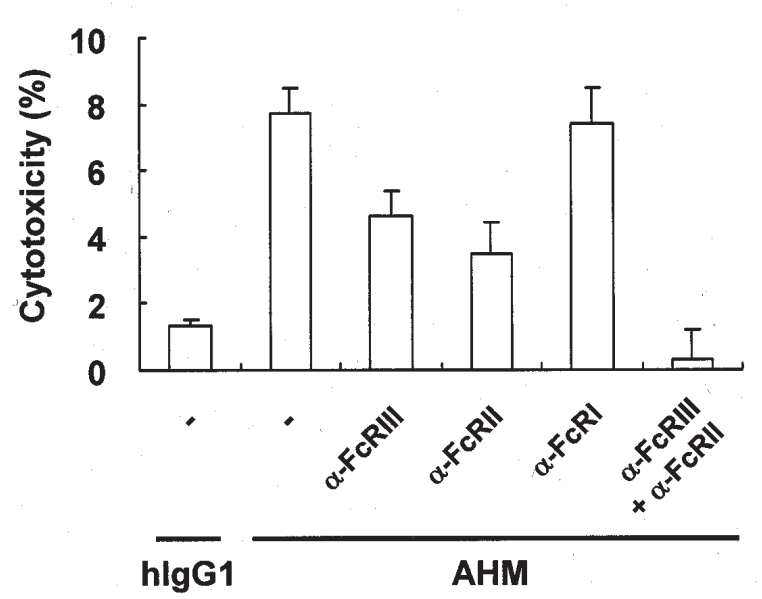

Figure 8. ADCC activity of AHM mediated by human effector cells. (A), PBMC was collected from peripheral blood of healthy volunteers. NK cells were depleted from PBMC by magnetic cell sorting. KPMM2 cells that had been labeled with ${ }^{51} \mathrm{Cr}$ were incubated with PBMC containing $(+\mathrm{NK})$ or not containing (-NK) NK cells for $4 \mathrm{~h}$ at an E:T ratio of 20 in the presence of $1 \mu \mathrm{g} /$ $\mathrm{ml} \mathrm{AHM} \mathrm{(white)} \mathrm{or} \mathrm{hIgG1} \mathrm{(grey).} \mathrm{(B),} \mathrm{NK} \mathrm{cells} \mathrm{were} \mathrm{purified} \mathrm{by} \mathrm{magnetic}$ cell sorting from PBMC collected from healthy volunteers. ADCC activity of AHM against KPMM2 cells was determined as in (A). Anti-Fc $\gamma$ RIII, II or I mAbs were added to the culture to give a final concentration of $10 \mu \mathrm{g} / \mathrm{ml}$ (FcRIII and II) or $20 \mu \mathrm{g} / \mathrm{ml}$ (FcRI) together with AHM. (C), Monocytes were purified by magnetic cell sorting from PBMC collected from healthy volunteers. ADCC activity of AHM against KPMM2 cells were determined as in (A) except for an E:T ratio of 50. Anti-Fc $\gamma$ RIII, II or I mAbs were added to the culture to give a final concentration of $10 \mu \mathrm{g} / \mathrm{ml}$ together with AHM.
Forum Proceedings Nov 3, 2003). Therefore, AHM may be safely combined with other drugs at full dose in clinical settings to further improve the efficacy.

\section{Acknowledgements}

We thank F. Ford for proofreading the manuscript.

\section{References}

1. Kyle RA: Multiple myeloma: review of 869 cases. Mayo Clin Proc 50: 29-40, 1975.

2. Klein B: Cytokine, cytokine receptors, transduction signals and oncogenes in human multiple myeloma. Semin Hematol 32: 4-19, 1995.

3. Kyle RA and Rajkumar SV: Multiple myeloma. N Engl J Med 351: 1860-1873, 2004.

4. Barlogie B, Shaughnessy J, Tricot G, et al: Treatment of multiple myeloma. Blood 103: 20-32, 2004.

5. Hideshima T, Bergsagel PL, Kuehl WM and Anderson KC: Advances in biology of multiple myeloma: clinical applications. Blood 104: 607-618, 2004.

6. Ohtomo T, Sugamata Y, Ozaki Y, et al: Molecular cloning and characterization of a surface antigen preferentially overexpressed on multiple myeloma cells. Biochem Biophys Res Commun 258: 583-591, 1999.

7. Goto T, Kennel SJ, Abe M, et al: A novel membrane antigen selectively expressed on terminally differentiated human B cells. Blood 84: 1922-1930, 1994.

8. Ozaki S, Kosaka M, Wakatsuki S, Abe M, Koishihara Y and Matsumoto T: Immunotherapy of multiple myeloma with a monoclonal antibody directed against a plasma cell-specific antigen, HM1.24. Blood 90: 3179-3186, 1997.

9. Ono K, Ohtomo T, Yoshida K, et al: The humanized antiHM1.24 antibody effectively kills multiple myeloma cells by human effector cell-mediated cytotoxicity. Mol Immunol 36: 387-395, 1999.

10. Ozaki S, Kosaka M, Wakahara Y, et al: Humanized antiHM1.24 antibody mediates myeloma cell cytotoxicity that is enhanced by cytokine stimulation of effector cells. Blood 93: 3922-3930, 1999.

11. Tejedor F and Hunter JPG: Iodination of biological samples without loss of functional activity. Anal Biochem 127: 143-149, 1975.

12. Goto H, Shimazaki C, Tatsumi T, et al: Establishment of a novel myeloma cell line KPMM2 carrying $t(3 ; 14)(\mathrm{q} 21 ; \mathrm{q} 32)$, which proliferates specifically in response to interleukin-6 through an autocrine mechanism. Leukemia 9: 711-718, 1995.

13. Tsunenari T, Koishihara Y, Nakamura A, et al: New xenograft model of multiple myeloma and efficacy of a humanized antibody against human interleukin-6 receptor. Blood 90: 2437-2444, 1997. 